\title{
Zum Pneumokokkenschutz reichen jetzt 3 Impfdosen
}

Die neuen STIKO-Empfehlungen sehen Änderungen beim Pneumokokkenschutz von Säuglingen und bei der Gelbfieberimpfung vor. Die Meningokokken-B-Impfung wird jetzt für Risikogruppen empfohlen.

Wichtigste Änderungen im neuen Impfkalender betreffen die PneumokokkenImpfung von Säuglingen. Für diese reichen künftig drei statt bisher vier Dosen eines Konjugatimpfstoffs aus. Das neue 2+1-Schema hat den Vorteil, dass Säuglinge im Alter von drei Monaten eine Impfung weniger benötigen. Neue Daten zur Wirksamkeit des kürzeren Impfschemas hatten die Änderung möglich gemacht. Für Frühgeborene wird unverändert das 3+1-Schema empfohlen (im Alter von 2, 3, 4 und 11-14 Monaten). Mit der
Pneumokokken-Impfung sollen Hospitalisierung, Behinderung und Tod infolge schwerer Erkrankungen reduziert werden. Die Zahl wird in Deutschland auf elf Fälle pro 100000 Kinder im Alter unter fünf Jahren geschätzt.

Der seit 2013 verfügbare Impfstoff gegen Meningokokken B wird jetzt Personen mit spezifischen Grundkrankheiten empfohlen (Indikationsimpfung). Die Entscheidung hierfür sollen Ärzte nach individueller Risikoabschätzung treffen. Bei der Gelbfieberimpfung empfiehlt die STIKO aufgrund der Änderungen in den internationalen Gesundheitsvorschriften (IGV) keine Auffrischimpfung mehr. Die Änderung wurde möglich, nachdem in den vergangenen Jahren mehrere Studien auf einen lebenslangen Impfschutz nach einmaliger Gelbfieberimpfung hingewiesen haben.
Einzelne Impftermine unbedingt einhalten!

Änderungen gibt es auch bei den Varizella-Zoster-Immunglobulinen. Diese sind bei fehlender Immunität gegen das Varizella-Zoster-Virus wichtig, wenn ein erhöhtes Risiko für eine schwere Windpockeninfektion besteht und nicht mit dem Lebendimpfstoff geimpft werden kann. Hier hat die STIKO ihre Empfehlung beim Personenkreis für die Impfung (neu: Frühgeborene mit Erreger-Kontakt) und dem Zeitraum der Anwendung (bis zehn Tage nach Erregerkontakt) erweitert. Für die bestmögliche Wirksamkeit müssen die einzelnen Impftermine unbedingt eingehalten werden.

(wg)

www.aerztezeitung.de
- krebs, die sich in der BI-RADS-Klassifikation niederschlagen, gibt es eine auf die Sonografie gestützte Malignitätsbeurteilung von Schilddrüsenknoten. Sie nennt sich TI-RADS, „Thyroid Imaging Reporting and Data System“. Verdächtig werden Knoten ab TI-RADS 4. In der Kategorie $4 \mathrm{~A}$ ist der Befund noch mäßig bedenklich, es fehlen hochverdächtige Merkmale: länger als breit, unregelmäßige Begrenzung, Mikrokalzifizierungen, sehr echoarm, sehr hart. Ab TI-RADS 4B beginnt die Zone der sehr malignitätsverdächtigen Befunde. Hier liegen bis zu zwei der genannten Merkmale vor. TI-RADS 5 schließlich bedeutet, dass mindestens drei Kriterien vorliegen und/oder verdächtige Lymphnoten zu erkennen sind.

Der positive Vorhersagewert der B-Mode-Sonografie dafür, dass die FNAZ ein Malignom ans Licht bringen werde, beträgt $6 \%$ für Knoten mit TI-RADS $4 \mathrm{~A}$, $69 \%$ bei TI-RADS 4B und $100 \%$ bei TIRADS 5. Durch Hinzunahme der Elastografie ändern sich die Werte auf $1,6 \%$, $35,9 \%$ und $100 \%$ [3]. „Durch diese standardisierte Erfassung sonografischer Malignitätskriterien können 52\% aller Schild- drüsenknoten mit einem falsch negativen Risiko von $0,3 \%$ als benigne klassifiziert werden und müssen nicht mit der FNAZ weiter abgeklärt werden", betont Paschke.

\section{Hoher Stellenwert klinischer \\ Kriterien}

Gemäß der Leitlinie ist die Feinnnadelaspiration ultraschallgestützt vorzunehmen, weil dies weniger nicht verwertbare und falsch negative Proben zur Folge hat. Auch ist es unter sonografischer Kontrolle leichter, Durchmesser und Länge der Nadel adäquat zu wählen. Unter optimalen Bedingungen werden laut Paschke 60-80\% der punktierten Knoten zytologisch als benigne klassifiziert, $10-20 \%$ als follikuläre Proliferation, 2,5-10\% als zytologisch suspekt und 3,5-10\% als zytologisch maligne. $10-15 \%$ der Punktate sind diagnostisch nicht verwertbar, die Biopsie muss dann wiederholt werden.

In den Händen erfahrener Untersucher ist die FNAZ die beste Methode, zwischen malignen und benignen Schilddrüsenknoten zu unterscheiden. Die Sensitivität erreicht $83 \%$, die Spezifität 92\%. „Eindeutige klinische Malignitätskriterien sollten aber immer einen höheren Stellenwert als (widersprüchlich) benigne FNAZ-Ergebnisse haben und gegebenenfalls allein zur Operation führen“, so Paschke.

\section{Somatische Mutationen und molekulare FNAZ}

Zusätzliche Aussagekraft wächst der (molekularen) FNAZ durch die Entdeckung somatischer Mutationen bei Schilddrüsenkarzinomen zu. Paschkes Ansicht nach wird sich dies besonders bei follikulären Neoplasien bzw. Proliferationen auswirken. Hier ist etwa bei Nachweis einer BRAF-Mutation eine totale Thyreoidektomie mit Lymphknotendissektion des zentralen Kompartiments angezeigt. Bei negativem Ergebnis der Mutationssuche können die Patienten in die Nachbeobachtung durch einen Spezialisten mit Wiederholung der FNAZ entlassen werden. Die Zahl diagnostischer Lobektomien könnte also sinken.

(Robert Bublak)

\footnotetext{
1. Paschke R. HNO Nachrichten 2014; 44 (2): 42-45; 2. Gharib Het al. Endocrine Practice 2010; 16 (Suppl 1): 1-43; 3. Russ $G$ et al. European Journal of Endocrinology 2013; 168: 649-655
} 